Volume 7, No. 12 December 2019

International Journal of Emerging Trends in Engineering Research

Available Online at http://www.warse.org/IJETER/static/pdf/file/ijeter157122019.pdf

https://doi.org/10.30534/ijeter/2019/157122019

\title{
Wavelet Coherence as a Tool for Visualizing the Relationship between Glomerular Filtration Rate and Renal Artery Blood Flow Velocity
}

\author{
Wadah M. Ali ${ }^{1,3}$, *Lana Haider Ahmed ${ }^{2,3}$, Iman A Babiker ${ }^{1}$, Albin Babu M Wilson ${ }^{1}$, Altahir Abdalmalik \\ Abukhiar ${ }^{1}$, M.E.M. Garelabi ${ }^{2,3}$, Hussien Abdelrhman Hussien ${ }^{4}$, Mayada A. Emak ${ }^{5}$, Vyacheslav \\ Lyashenko $^{6}$, Mohamed M Abuzaid ${ }^{7}$ \\ ${ }^{1}$ Department Medical Imaging, College of Health Science, Gulf Medical University, Ajman, UAE, \\ dr.wadah@gmu.ac.ae \\ ${ }^{2}$ Collage of medical radiological science, Sudan University of Science and Technology, Sudan, \\ Lana.Haider22@yahoo.com \\ ${ }^{3}$ Faculty of Radiological and Nuclear Medicine Science, National Ribat University. \\ ${ }^{4}$ Faculty of Radiography and Medical Imaging, National University Sudan, Sudan, Hdinar@ NU.edu.sd \\ ${ }^{5}$ Nuclear Medicine Unit, Medical Imaging Department, Royal Care International Hospital, Khartoum, Sudan \\ ${ }^{6}$ Department of Informatics, Kharkiv National University of RadioElectronics, Ukraine, \\ lyashenko.vyacheslav@gmail.com \\ ${ }^{7}$ Assistant Professor, Department of Medical Imaging, University of Sharjah, Sharjah, United Arab Emirates. \\ mabdelfatah@sharjah.ac.ae
}

\begin{abstract}
The study of glomerular filtration rate using renal scintigraphy with enhancement and blood flow velocity in renal artery has been carried. We estimated the relationship between glomerular filtration rate and renal artery blood flow rate for each kidney and their mutual influence. To explain the results of the relationship and visualization of the research process, we used wavelet coherence. The expediency of using the wavelet methodology for conducting intellectual diagnostics for the detection of kidney diseases is shown.
\end{abstract}

Key words: Glomerular Filtration Rate, Renal Artery Blood Flow Velocity, Kidney Diagnostics, Wavelet Analysis, Visualization, Data Mining

\section{INTRODUCTION}

Any phenomenon or process can be represented as some data set [1], [2]. The analysis of such data helps to obtain new data, new knowledge. New information helps to understand and explain the phenomena and processes that are being investigated. It also helps to make the right decisions.

Of particular importance is the analysis of data in medicine [3], [4]. This is due to the fact that we are considering data that characterize the health and possible diseases of patients. Therefore, it is important to know more information than primary data.

An example of data analysis in medicine is the diagnosis of kidney disease [5]. The radionuclide evaluation of renal function has been introduced since 1950. Early studies used a radioactive material with external probe to produce time activity curve to show the uptake and excretion of the radiopharmacutical within the kidneys [6]. The application of radionuclide procedure has proved to be effective at evaluation of renal disorders.

At the same time, it is also necessary to use various simple procedures for quick analysis of primary data. This will help to conduct a quick diagnosis of kidney disease, provide the necessary assistance to the patient. Applying different approaches to data analysis will also help to understand the dynamics of processes that affect the development of relevant diseases. At the same time, visualization of the research process is also important. In general, this determined the subject of this work.

\section{MATERIALS AND METHODS}

2.1 Overview of data analysis tools in the diagnosis of kidney

One of the main tools for analyzing various data are statistical analysis methods. These methods are also used in the study of kidney disease.

For example, in [7], [8], an analysis is made of various data that are characteristic of kidney disease. Such an analysis is based on the methods of descriptive and comparative statistics, clustering data on various groups of patients. Various diagrams and tables are also used, which allow to generalize and systematize the results of the study. 
Wadah M. Ali et al., International Journal of Emerging Trends in Engineering Research, 7(12), December 2019, 818 - 823

A. Phrommintikul, S. J. Haas, M. Elsik, and H. Krum use meta-analysis to analyze kidney disease [9]. Such an analysis involves the selection of certain markers for the corresponding study. Next, correlation analysis is used to determine the factors of mutual influence between different markers. As visualization methods, various charts and graphs are used.

The study [10] is based on cluster and factor analysis. This allowed us to process large amounts of data and draw conclusions about kidney diseases for each group of patients. To visualize the results, classic clustering diagrams were used.

In [11], an analysis was made of the comparative effectiveness of various markers in the diagnosis of kidney diseases. This allows you to determine the group of markers that are appropriate to use in the study of various kidney diseases. However, such an analysis does not use data visualization methods that help interpret the results.

V. Kunwar, K. Chandel, A. S. Sabitha and A. Bansal perform intelligent data analysis [12]. This allows you to classify groups of patients according to the type of kidney disease.

Thus, classic methods for the analysis of primary data are used to diagnose kidney diseases. To visualize the results of the analysis, graphs, charts, tables are used.

\subsection{Wavelet Coherence as a Data Analysis Tool}

Wavelet methodology is one of the methods of data processing. Using the wavelet methodology, you can process various data sets [13], [14]. For such an analysis, it is necessary that the data set has a structure that is similar to the structure of a time series. Any data set can be converted to a data set that has a time series structure. It is necessary to select a certain data set and to rank all the data for the selected data set [15].

To analyze the relationships between different data sets, wavelet coherence can be used [15], [16]. This analysis is based on cross-references. To do this, use the following formula [16], [17]:

$$
R^{2}\left(k, k_{l}\right)=\frac{\left|\Theta\left(k_{l}^{-1} \Delta_{x y}^{g}\left(k, k_{l}\right)\right)\right|}{\Theta\left(k_{l}^{-1}\left|\Delta_{x}^{g}\left(k, k_{l}\right)\right|^{2}\right) \Theta\left(k_{l}^{-1}\left|\Delta_{y}^{g}\left(k, k_{l}\right)\right|^{2}\right)},
$$

where:

$\Theta-$ is a smoothing operator,

$k$ and $k_{l}$ - data groups, which are investigated;

$$
\begin{aligned}
& g \text { - data group for ranking; } \\
& \Delta\left(k, k_{1}\right)-\text { cross wavelet spectra for different time series } \\
& z \text { and } z_{1}, \\
& x \text { - data number in the test series, } \\
& y \text { - characterizes the depth of cross-references, } \\
& 0 \leq R^{2}\left(z, z_{1}\right) \leq 1 .
\end{aligned}
$$

Wavelet coherence may provide an explanation for the various correlations between the data being investigated. This is due to the fact that we are considering cross-references for different depths of the source data.

\subsection{Data for analysis}

We are considering a group of 210 patients who have different kidney diseases. In this group there were 99 male and 111 female aged 9-76 years. These data were obtained at a radiological center in Sudan.

As markers of liver disease, we consider: glomerular filtration rate and renal artery blood flow velocity.

Glomerular filtration rate (PSV) may be a marker of renal function, as this marker is useful for quantifying and grade stenosis. At the same time, renal stenosis directly affects renal function - the speed of blood flow in the renal artery (GFR).

Measurement of glomerular filtration rate is also considered an important parameter in the diagnosis of renal function and early monitoring of renal dysfunction.

For analysis, we use tools such as regression and correlation analysis, wavelet coherence, comparative analysis.

\section{RESULTS AND DISCUSSION}

\subsection{Results of regression and correlation analysis}

Figure 1 showed the relationship between the PSV $(\mathrm{cm} / \mathrm{sec})$ and GFR $(\mathrm{ml} / \mathrm{min})$ of the right kidneys. We see that this relationship is proportionally dependent. This relationship is described by the equation (significance level 0.85 ):

$$
y=1.889 x-5.005,
$$

where:

$$
x \text { - values PSV for the right kidney, }
$$$$
y \text { - values GFR for the right kidney. }
$$ 


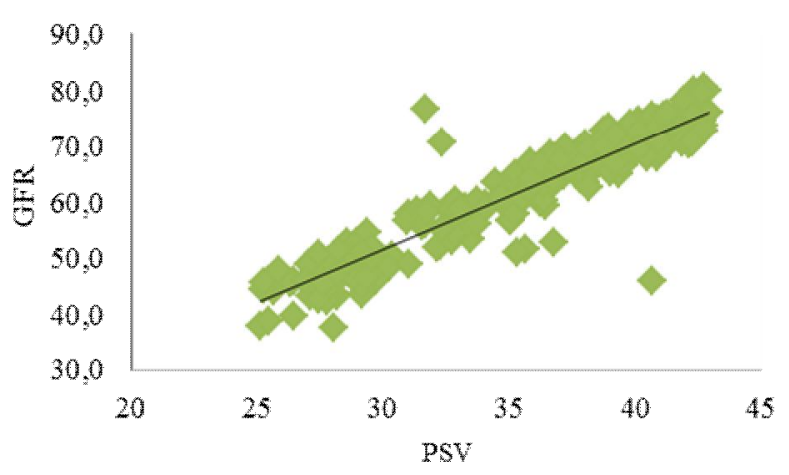

Figure 1: Relationship between PSV and GFR for the right kidney

Figure 3 showed the relationship between the PSV and GFR of the left kidneys. This relationship is described by the equation (significance level 0.75):

$$
y=1.801 x-4.153,
$$

where:

$$
x \text { - values PSV for the left kidney, }
$$$$
y \text { - values GFR for the left kidney. }
$$

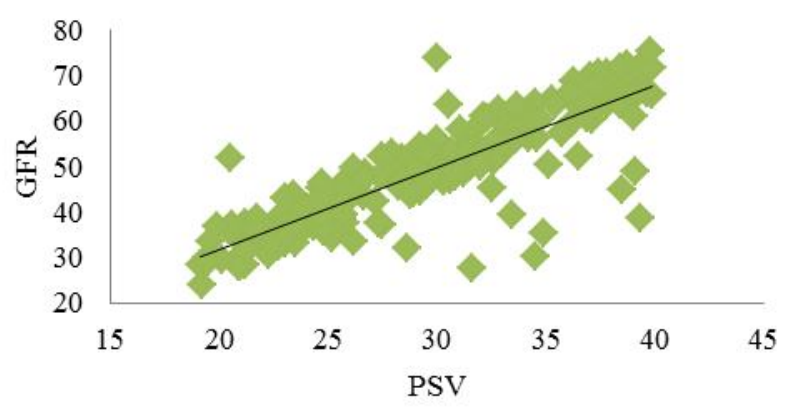

Figure 2: Relationship between PSV and GFR for the left kidney

The figures also showed that there was minimum difference between the right and left kidneys concerning the relation between the PSV and GFR.

It should also be noted that the relationship between PSV and GFR for the right kidney is more significant than for the left kidney.

Figure 3 showed the relationship between the PSV for the right kidney and total GFR. This relationship is described by the equation (significance level 0.46 ):

$$
y=2.283 x+30.08,
$$

where:

$$
\begin{aligned}
& x \text { - values PSV for the right kidney, } \\
& y \text { - total values GFR. }
\end{aligned}
$$

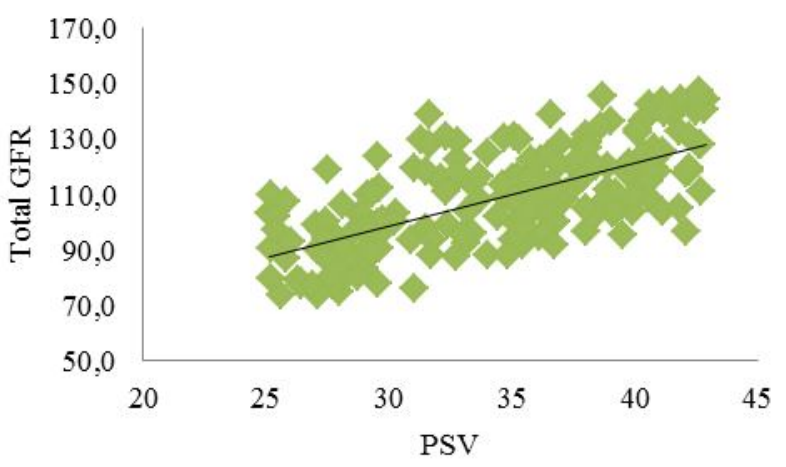

Figure 3: Relationship between PSV for the right kidney and total GFR

We also see that Relationship between PSV for the right kidney and total GFR is lower, than for the data of Figure 1 and Figure 2. This is due to the fact that we are considering the generalized value of GFR.

Figure 4 showed the relationship between the PSV for the left kidney and total GFR. This relationship is described by the equation (significance level 0.46 ):

$$
y=1.949 x+51.90,
$$

where:

$x$ - values PSV for the left kidney, $y$ - total values GFR.

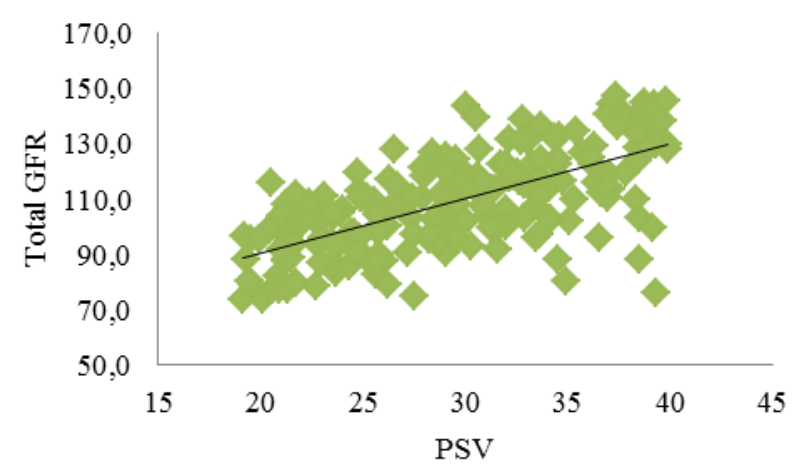

Figure 4: Relationship between PSV for the left kidney and total GFR

The figures also showed that there was significant difference between the right and left kidneys concerning the relation between the PSV and total GFR.

It should also be noted that the results correlate with classical approaches that are used in the diagnosis of kidney disease [18]-[20]. At the same time, it must be emphasized that in such studies, two series of data are analyzed. However, the issues of the relationship of the observed correlation depending on other parameters are not considered. 
In this context, for example, it is necessary to consider the relationship between the glomerular filtration rate and the blood flow velocity in the renal artery for different age groups. This will help to understand the presence of appropriate levels of significance between the studied markers, to expand the boundaries of the study.

\subsection{Wavelet Coherence Results}

Consider the wavelet coherence between the glomerular filtration rate and the blood flow velocity in the renal artery in accordance with the data in Figure 1 - Figure 4. Consider this relationship with respect to the age of the patients.

The figures below show the results of wavelet coherence. These figures show:

along the axis $x$ of the patient age value. These values are represented by serial numbers in accordance with the number of patients in the sample (210 patients). The smallest serial number corresponds to the patient's age of 9 years, the largest - 76 years;

along the axis $y$ - the depth of the relationship between the studied data values, for which we determine the values of the wavelet coherence. This depth also displays various analysis intervals for patient age values;

the dashed white line limits the region of reliable values of wavelet coherence (with a confidence level of at least 0.95). These values are inside the dashed line;

the figure also shows a scale for analyzing the significance of wavelet coherence data. The value of wavelet coherence is in the range from 0 to 1 . Such data for clarity also have color values. Separate areas are localization of consistency (the significance of wavelet coherence tends to 1) or inconsistency of the data (significance of wavelet coherence, usually 0 ), which are being studied.

Figure 5 shows the wavelet coherence between the PSV and GFR for the right kidney. These values are shown according to the age of the patients.

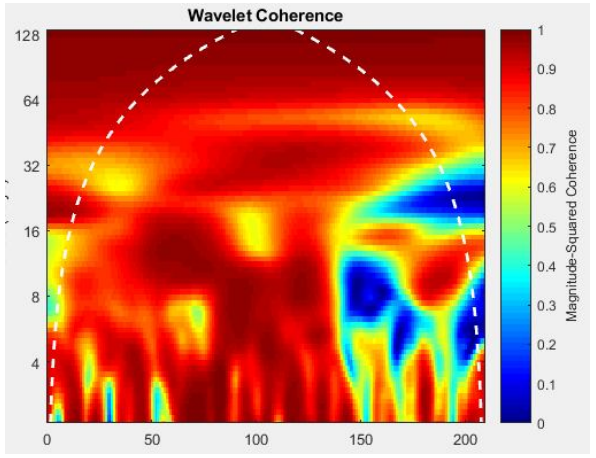

Figure 5: Wavelet coherence between PSV and GFR for the right kidney

We see that data between PSV and GFR for the right kidney is consistent. Such consistency is observed for almost all age categories of patients. This consistency can explain the high level of significance (significance level 0.85) for relationship between the PSV and GFR right kidney (see Figure 1). The smallest consistency relationship between the PSV and GFR for the right kidney can be seen for the age category of patients.

Figure 6 shows the wavelet coherence between the PSV and GFR for the left kidney. These values are also shown according to the age of the patients.

We can see less consistency between PSV and GFR for the left kidney (see Figure 2), compared with the data in Figure 5. This can explain the lower significance level between PSV and GFR for the left kidney (see Figure 2), compared with the data in Figure 1 (significance level between PSV and GFR for the right kidney). It should also be noted that for younger patients, the consistency between PSV and GFR for the left kidney is less. This can explain the difference between the data in Figure 1 and Figure 2.

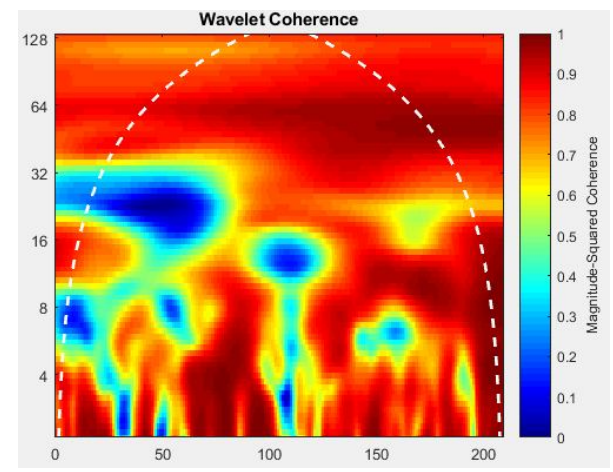

Figure 6: Wavelet coherence between PSV and GFR for the left kidney

Figure 7 shows the wavelet coherence between PSV for the right kidney and total GFR. 


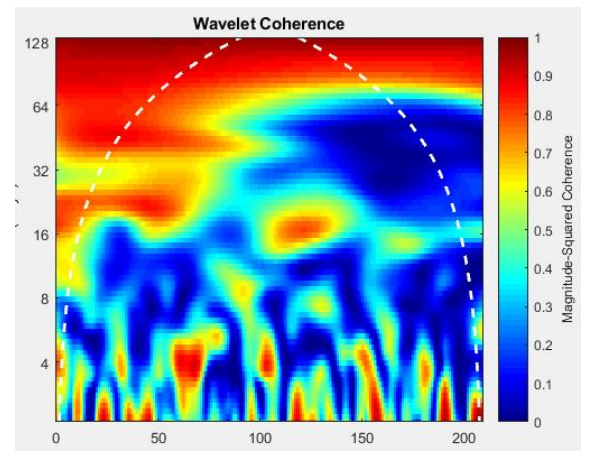

Figure 7: Wavelet coherence between PSV for the right kidney and total GFR

Figure 8 shows the wavelet coherence between PSV for the left kidney and total GFR.

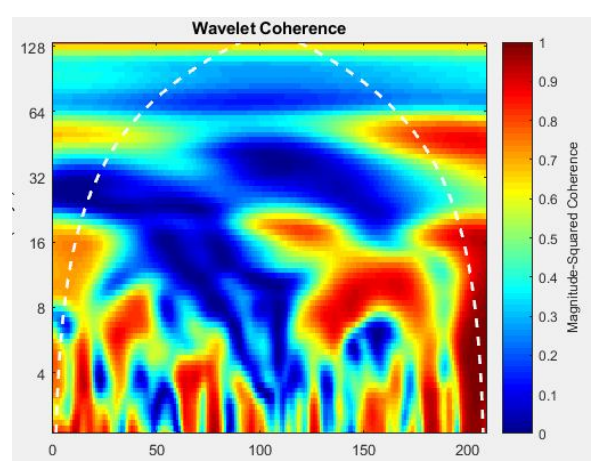

Figure 8: Wavelet coherence between PSV for the left kidney and total GFR

We see that the consistency between PSV for the left kidney and total GFR (see Figure 7) and between PSV for the left kidney and total GFR (see Figure 8) is significantly less than for the data in Figure 5 and Figure 6. This can explain the corresponding the significance level for the data in Figure 3 and Figure 4.

It should also be noted that there is a different agreement between the data in Figure 7 and Figure 8. This explains the different dynamics of the data for Figure 3 and Figure 4, in their comparison. The data consistency for Figure 8 is greater than for Figure 7.

Thus, wavelet coherence is a tool that helps to explain and understand the relationships between the markers studied in the diagnosis of kidneys. This fact distinguishes this work from the classical studies that were carried out in articles [12], [18]-[20].

\section{CONCLUSION}

Qualitative diagnosis of the kidneys is the basis for the timely treatment of the disease. We examined the relationship between glomerular filtration rate and renal arterial blood flow velocity. For analysis, we used the wavelet methodology.
We specified the relationship between glomerular filtration rate and renal arterial blood flow velocity for different age groups of patients. This allowed us to explain the existing meanings of the relationships between the various markers that we are considering. Wavelet coherence also allows visualization for the process under study.

\section{REFERENCES}

1. R. C. Gustilo, and C. C. Escolar-Jimenez. An Analytic Hierarchy Process Approach in the Shortlisting of Job Candidates in Recruitment, International Journal of Emerging Trends in Engineering Research, Vol. 7, no. 9, pp. 333-339, 2019. https://doi.org/10.30534/ijeter/2019/17792019

2. C. C. Escolar-Jimenez, K. Matsuzaki, , K. Okada, R. C. Gustilo. Data-Driven Decisions in Employee Compensation utilizing a Neuro-Fuzzy Inference System, International Journal of Emerging Trends in Engineering Research, Vol. 7, no. 8, pp.163-169, 2019. https://doi.org/10.30534/ijeter/2019/10782019

3. P. Orobinskyi, D. Petrenko, and V. Lyashenko. Novel Approach to Computer-Aided Detection of Lung Nodules of Difficult Location with Use of Multifactorial Models and Deep Neural Networks, in 15th International Conference on the Experience of Designing and Application of CAD Systems (CADSM), 2019, pp. 1-5. https://doi.org/10.1109/CADSM.2019.8779340

4. A. Rabotiahov, O. Kobylin, Z. Dudar, and V. Lyashenko. Bionic image segmentation of cytology samples method, In International Conference on Advanced Trends in Radioelecrtronics, Telecommunications and Computer Engineering (TCSET), 2018, pp. 665-670. https://doi.org/10.1109/TCSET.2018.8336289

5. M. Ketteler, and et al.. Diagnosis, evaluation, prevention, and treatment of chronic kidney disease-mineral and bone disorder: Synopsis of the kidney disease: improving global outcomes 2017 clinical practice guideline update, Annals of internal medicine, Vol. 168, no. 6, pp. 422-430, 2018. https://doi.org/10.7326/M17-2640

6. Y. Pei, and et al.. Imaging-based diagnosis of autosomal dominant polycystic kidney disease, Journal of the American Society of Nephrology, Vol. 26, no. 3, pp. 746-753, 2015. https://doi.org/10.1681/ASN.2014030297

7. K. T. Mills, and et al.. A systematic analysis of worldwide population-based data on the global burden of chronic kidney disease in 2010, Kidney international, Vol. 88, no. 5, pp. 950-957, 2015. https://doi.org/10.1038/ki.2015.230

8. D. E. Weiner, and et al.. Chronic kidney disease as a risk factor for cardiovascular disease and all-cause mortality: a pooled analysis of community-based studies, Journal of the American Society of Nephrology, 
Vol. 15, no. 5, pp. 1307-1315, 2004. https://doi.org/10.1097/01.ASN.0000123691.46138.E2

9. A. Phrommintikul, S. J. Haas, M. Elsik, and H. Krum. Mortality and target haemoglobin concentrations in anaemic patients with chronic kidney disease treated with erythropoietin: a meta-analysis, The lancet, Vol. 369, no. 9559, pp. 381-388, 2007. https://doi.org/10.1016/S0140-6736(07)60194-9

10. E. R. Seaquist, F. C. Goetz, S. Rich, and J. Barbosa. Familial clustering of diabetic kidney disease, New England Journal of Medicine, Vol. 320, no. 18, pp. 1161-1165, 1989. https://doi.org/10.1056/NEJM198905043201801

11. S. C. Palmer, and et al.. Comparative efficacy and safety of blood pressure-lowering agents in adults with diabetes and kidney disease: a network meta-analysis, The Lancet, Vol. 385, no. 9982, pp. 2047-2056, 2015. https://doi.org/10.1016/S0140-6736(14)62459-4

12. V. Kunwar, K. Chandel, A. S. Sabitha, and A. Bansal. Chronic Kidney Disease analysis using data mining classification techniques, in 6th International Conference-Cloud System and Big Data Engineering, 2016, pp. 300-305. https://10.1109/CONFLUENCE.2016.7508132

13. V. Lyashenko, O. Kobylin, and Y. Baranchykov. Ideology of Image Processing in Infocommunication Systems, in International Scientific-Practical Conference Problems of Infocommunications. Science and Technology (PIC S\&T), 2018, pp. 47-50. https://doi.org/10.1109/INFOCOMMST.2018.8632124

14. V. Lyashenko, O. Kobylin, and M. Minenko. Tools for Investigating the Phishing Attacks Dynamics, in International Scientific-Practical Conference Problems of Infocommunications. Science and Technology (PIC S\&T), 2018, pp. 43-46. https://doi.org/10.1109/INFOCOMMST.2018.8632100

15. V. Lyashenko, O. Zeleniy, S. K. Mustafa, and M. A. Ahmad. An Advanced Methodology for Visualization of Changes in the Properties of a Dye, International Journal of Engineering and Advanced Technology, Vol. 9, no. 1, pp. 711-7114, 2019. https://doi.org/10.35940/ijeat.A1496.109119

16. C. Torrence, and P. J. Webster. Interdecadal changes in the ENSO-monsoon system, Journal of Climate, Vol. 12 , no. 8, pp. 2679-2690, 1999.

https://doi.org/10.1175/1520-0442(1999)012<2679:ICI TEM>2.0.CO;2

17. A. Grinsted, J. C. Moore, and S. Jevrejeva. Application of the cross wavelet transform and wavelet coherence to geophysical time series, Nonlinear processes in geophysics, Vol. 11, no. 5/6, pp. 561-566, 2004.

18. T. Namikoshi, and et al.. Relationship between vascular function indexes, renal arteriolosclerosis, and renal clinical outcomes in chronic kidney disease, Nephrology, Vol. 20, no. 9, pp. 585-590, 2015. https://doi.org/10.1111/nep.12483
19. R. R. Townsend, and et al.. Association of pulse wave velocity with chronic kidney disease progression and mortality: findings from the CRIC study (chronic renal insufficiency cohort), Hypertension, Vol. 71, no. 6, pp. 1101-1107, 2018. https://doi.org/10.1161/HYPERTENSIONAHA.117.106 48

20. S. Kawakami, and et al.. Association between exercise intensity and renal blood flow evaluated using ultrasound echo, Clinical and experimental nephrology, Vol. 22, no. 5, pp. 1061-1068, 2018. https://doi.org/10.1007/s10157-018-1559-1 